\title{
Cost-Utility Analysis of Nivolumab in Adjuvant Treatment of Melanoma in France
}

\author{
Bruno Bregman · Siguroli Teitsson • Isabella Orsini • François-Emery Cotté • \\ Adenike Amadi · Andriy Moshyk · Stéphane Roze · Anne-Françoise Gaudin
}

Received: July 15, 2020 / Published online: September 13, 2020

(C) The Author(s) 2020

\begin{abstract}
Introduction: The aim of the current study is to estimate the cost-effectiveness of adjuvant treatment with nivolumab relative to clinically relevant comparators in adult patients with melanoma with lymph node involvement or metastatic disease who have undergone complete resection from a French societal perspective.

Methods: The comparators were observation, low-dose interferon and pembrolizumab. A subgroup analysis was carried out in patients
\end{abstract}

Digital Features To view digital features for this article go to https://doi.org/10.6084/m9.figshare.12911369.

Electronic supplementary material The online version of this article (https://doi.org/10.1007/s13555020-00446-z) contains supplementary material, which is available to authorized users.

B. Bregman $(\bowtie)$ · F.-E. Cotté · A.-F. Gaudin Bristol-Myers Squibb, Rueil-Malmaison, France e-mail: bruno.bregman@bms.com

S. Teitsson · I. Orsini

Parexel International, London, UK

A. Amadi

Bristol-Myers Squibb, Uxbridge, UK

A. Moshyk

Bristol-Myers Squibb, Princeton, NJ, USA

S. Roze

HEVA-HEOR, Lyon, France with $B R A F$ mutation, adding dabrafenib plus trametinib. A three-state partitioned survival model was developed to project costs and health benefits over a 20-year time horizon. Extrapolation for recurrence-free survival (RFS) and overall survival (OS) was carried out using spline-based models. Because of the immaturity of OS data in pivotal trials for nivolumab and pembrolizumab, a predictive model of OS treatment effect based on RFS effect was developed using a correlation equation. Health state utilities and adverse events disutilities were derived from the CheckMate 238 trial and literature. Costs were estimated in 2019 euros. The model's primary outcome was efficiency frontier. Deterministic and probabilistic sensitivity analyses were conducted to assess the robustness of results.

Results: Observation, low-dose interferon and nivolumab were on the efficiency frontier. The incremental cost-utility ratio of nivolumab versus low-dose interferon (closest therapy on the efficiency frontier) was $€ 37,886 /$ quality-adjusted life year (QALY). Probabilistic sensitivity analysis reported an $80 \%$ probability of nivolumab being a cost-effective strategy for a willingness-to-pay threshold of $€ 52,000 / Q A L Y$. In the subgroup with $B R A F$ mutation, the efficiency frontier was not changed by the addition of dabrafenib plus trametinib.

Conclusions: Nivolumab is a cost-effective strategy as adjuvant treatment in adult patients with surgically resected melanoma in France. 
Keywords: Adjuvant melanoma; Costeffectiveness; France; Nivolumab

\section{Key Summary Points}

From a French societal perspective, nivolumab is a cost-effective treatment option in adult patients with melanoma with lymph node involvement or metastatic disease who have undergone complete resection.

Nivolumab was found on the efficiency frontier with observation and low-dose interferon. The incremental cost-utility ratio of nivolumab versus low-dose interferon was $€ 37,886 /$ quality-adjusted life year.

There was an $80 \%$ probability of nivolumab being a cost-effective strategy for a willingness-to-pay threshold of $€ 52,000 / q u a l i t y$-adjusted life year.

\section{DIGITAL FEATURES}

This article is published with digital features to facilitate understanding of the article. You can access the digital features on the article's associated Figshare page. To view digital features for this article go to https://doi.org/10.6084/m9. figshare.12911369.

\section{INTRODUCTION}

Melanoma is an aggressive form of cancer with poor prognosis. According to the World Health Organization, 132,000 new cases of melanoma are diagnosed annually worldwide [1]. In France alone, more than 15,000 new cases of melanoma were diagnosed in 2017, with an estimated 1783 deaths [2]. Melanoma is usually diagnosed at an early stage [3]. The severity of the disease depends on its evolution and its metastatic potential. In patients who present a nodal involvement (stage III), there is a high probability of developing distant metastases [4].

The standard treatment for melanoma is surgical resection. After surgery, few patients receive adjuvant treatment as limited therapeutic options are available. One available option is interferon, which has modest efficacy and is associated with dose-limiting toxicity, including flu-like syndrome $[5,6]$. Immune checkpoint inhibitors and targeted therapies have recently demonstrated efficacy as adjuvant therapies for patients with resected high-risk melanoma [7-11]. Among these therapies, the programmed death-1 (PD-1) inhibitors nivolumab, for resected stage III/IV melanoma, and pembrolizumab, for resected stage III melanoma, were approved by the European Medicines Agency (EMA) in 2018 based on recurrencefree survival (RFS) benefits in phase 3 trials $[12,13]$. Dabrafenib plus trametinib, a BRAF/ MEK inhibitor combination therapy, was approved by the EMA in 2019 for resected stage III melanoma with BRAF V600 mutation [14].

In the CheckMate 238 trial (NCT02388906) [11], nivolumab demonstrated significant improvement in the primary endpoint of RFS versus ipilimumab [hazard ratio (HR) $0.66,95 \%$ confidence interval (CI) 0.54-0.81] after an interim follow-up of at least 24 months. However, the secondary endpoint of overall survival (OS) was immature for an assessment after the 24-month follow-up.

It will be critical for health technology assessment agencies to estimate the clinical and economic value of nivolumab within the process of reimbursement decision. Whilst nivolumab cost-effectiveness in the treatment of advanced melanoma has been the subject of several trials [15-18], little information has been published on its cost-effectiveness as adjuvant treatment.

The objective of this analysis is to estimate the cost-effectiveness of nivolumab versus clinically relevant comparators in adult patients with melanoma with lymph node involvement or metastatic disease who have undergone complete resection from a French societal perspective, in compliance with the methodological guidelines published by the Haute Autorite de Santé (HAS) in France [19]. The analysis 
followed the guideline recommendations published by the Commission d'Évaluation Économique et de Santé Publique (CEESP) [20, 21]. The required outcome criteria for the reference case were an efficiency frontier and the calculation of an incremental cost-effectiveness ratio for non-dominated interventions, as reported previously $[22,23]$. The analysis estimated nivolumab cost-utility in the overall population and in a subgroup of patients with $B R A F$ mutation.

\section{METHODS}

\section{Population of Analysis}

Two populations were considered in the analysis: the overall population corresponding to the patients enrolled in the CheckMate 238 trial [11] and a subgroup consisting of patients with $B R A F$ mutation.

\section{Comparators}

Based on clinical recommendations [24, 25], approved indications and clinical practice in France $[26,27]$, the comparators for the overall population were nivolumab, observation, lowdose interferon and pembrolizumab. Dabrafenib plus trametinib was also included in the subgroup with BRAF mutation. Ipilimumab, which was approved in the USA as adjuvant treatment based on the European Organisation for Research and Treatment of Cancer (EORTC) 18071 trial [7], was not selected as it was not approved in the EU at the time of analysis, but was used as connecting therapy in indirect comparisons.

\section{Model Overview}

A three-state partitioned survival model was developed to evaluate the cost-utility of nivolumab relative to the comparators. The model was developed in Microsoft Excel 2016. The Visual Basic code was used to execute iterations needed for probabilistic sensitivity analysis (PSA) and deterministic sensitivity analysis
(DSA). The three distinct and mutually exclusive states were RFS, post-recurrence survival (PRS) and death, which represent the main stages of the disease in adult patients with resected melanoma. Thus, patients enter the model after complete resection (the RFS state), from which they can either enter the PRS state or the absorbing state (death). The proportion of patients in the PRS state was derived as the difference between the OS and RFS curves at each time point (Fig. 1). This approach was used to calculate the area under the RFS curves based on data from EORTC 18071 [7] and extrapolated over a maximum 20-year time horizon. After selection of a base case distribution model for extrapolating RFS and OS, which was chosen by the best fitting (statistical fit) survival distribution, HRs of indirect comparisons were then applied to generate survival extrapolation curves for comparators using observation as reference treatment.

The proportions of patients in each health state were calculated with regular 28-day (4 week) cycles, consistent with the various frequencies of administration of comparators, over the time horizon of 20 years. A half-cycle correction was also applied to improve the precision of estimations in each health state.

\section{Systematic Literature Review and Network Metaanalysis (NMA)}

A systematic literature review was conducted with the objective of identifying randomized clinical trials evaluating efficacy, safety and quality of life of adjuvant treatments for melanoma in patients who had undergone complete resection. An NMA was then performed to estimate the HRs for RFS and OS. The NMA of reported HRs assumed proportional hazards between treatments and was performed using a regression model with a contrast-based normal likelihood for the log HR of each trial in the network. The analyses included fixed and timevarying effects. The description of how the HRs from the NMA were used in the model is in section "Survival Extrapolation". 


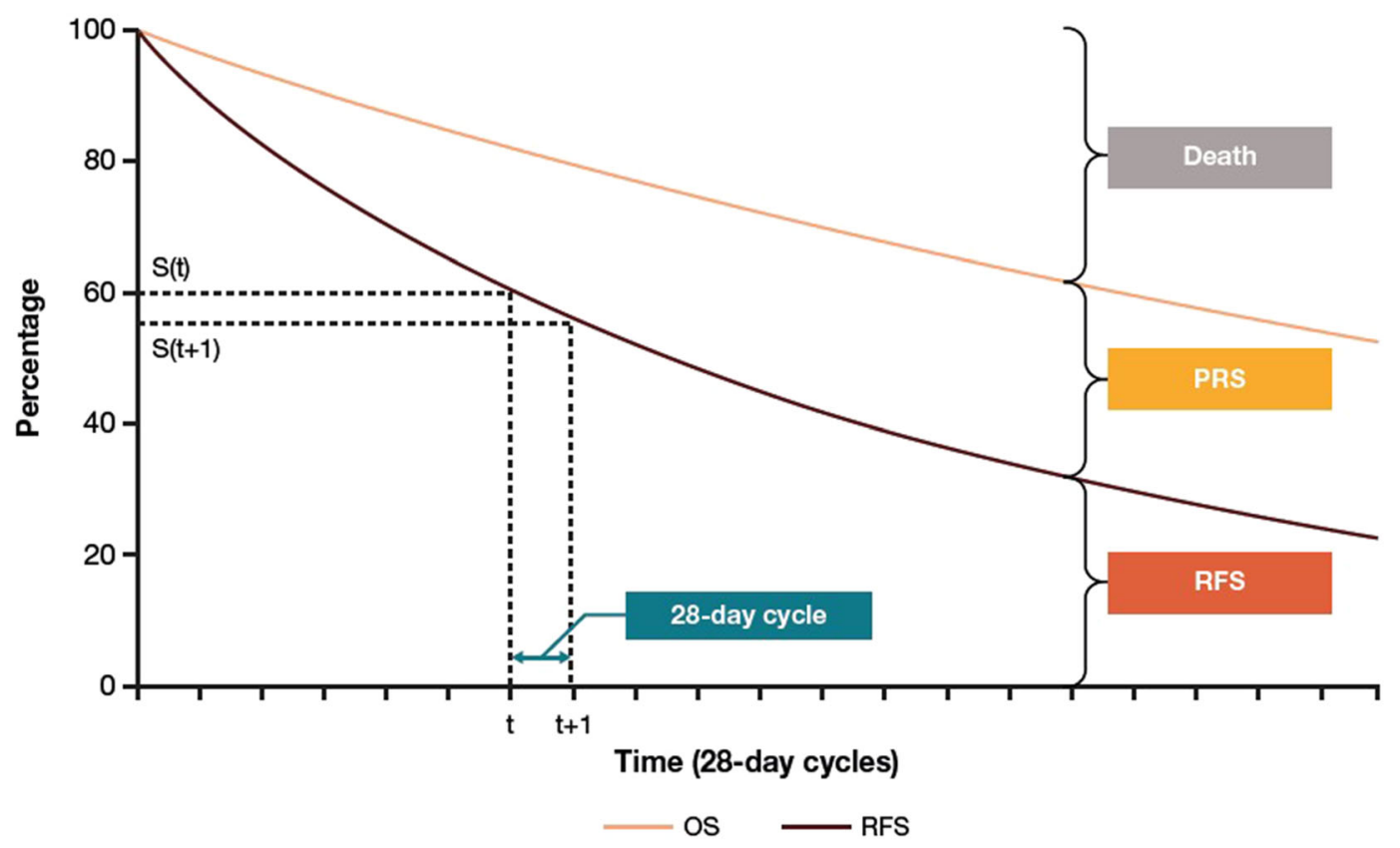

Fig. 1 Graphical representation of three-state survival model. OS overall survival, PFS post-recurrence survival, RFS recurrence-free survival, $S$ survival function (survival \% against time), $t$ time

\section{Modelling RFS to OS in CheckMate 238}

In the absence of OS data at the 24-month follow-up in CheckMate 238, a predictive model of OS using RFS was developed for the adjuvant treatment of melanoma [28, 29]. This model used the technique developed by Burzykowski et al. [30, 31], which enables calculation of a predictive $\mathrm{HR}_{\mathrm{OS}}$ (nivolumab versus observation) using the $\mathrm{HR}_{\mathrm{RFS}}$ (nivolumab versus observation) estimated by the NMA. The same method was used to generate $\mathrm{HR}_{\mathrm{OS}}$ (pembrolizumab versus observation). A literature review of trials of adjuvant treatment in melanoma that reported both RFS and OS was used to populate the model. A linear regression model was fitted through the treatment effects on RFS and OS estimated with adjustment for the measurement error of these estimates and was used to develop the following correlation equation:

$$
\mathrm{HR}_{\mathrm{OS}}=\exp .\left(-0.0049+0.7925 \times \log \left(\mathrm{HR}_{\mathrm{RFS}}\right)\right) \text {. }
$$

The $H_{R F S}$ was entered into the above correlation equation to estimate the final $\mathrm{HR}_{\mathrm{OS}}$ for nivolumab and pembrolizumab versus observation. The correlation equation developed by Siciu et al. [29] successfully predicted the $\mathrm{HR}_{\mathrm{OS}}$ of ipilimumab versus placebo in EORTC 18071 [7].

\section{Survival Extrapolation}

The observation (reference), RFS and OS curves were informed by the placebo arm in EORTC 18071 [7]; this trial had a minimum follow-up of 5.3 years, during which $68 \%$ of patients had recurrence events and $45 \%$ of patients died. Standard parametric and spline distributions were tested and fitted to the observation curves using Akaike information criterion (AIC)/Bayesian information criterion (BIC) statistics. The selection method of parametric distribution used the technical guideline developed for the National Institute for Health and Care Excellence [32]. The distributions offering optimal fit with the observation curves were spline twoknots hazards for RFS and spline one-knot normal for OS. HRs from indirect comparisons were 
then applied to generate RFS extrapolation curves for all comparators. HRs from indirect comparisons were applied to generate OS extrapolation curves for comparators having OS data (i.e. low-dose interferon and dabrafenib plus trametinib for the $B R A F$ mutation subgroup). For nivolumab and pembrolizumab, the $\mathrm{HR}_{\mathrm{O}}$ versus observation was obtained by the predictive correlation equation and applied to the observation OS curve.

\section{Other Inputs}

Information on other inputs including costs, utilities and adverse events are summarized in the Online Resource and Online Resources Tables 1-6. An annual discount rate of $4 \%$ was applied in the model for costs and health outcomes, as per HAS guidelines [19].

\section{Model Output}

The primary outcome of the model was the efficiency frontier; as described by Caro et al. [33], the frontier was presented in an efficiency plot with costs on the horizontal axis and value of benefits on the vertical axis. The efficiency frontier links interventions that are not dominated. The HAS guidelines [19] mentioned that 'health interventions on the efficiency frontier are identified as not dominated by an alternative (strict dominance) or combination of alternatives (extended dominance). Interventions are ranked in terms of costs (from the cheapest to the most expensive). Incremental cost-effectiveness ratios (ICERs) are then calculated for each intervention, compared with the next most-expensive, non-dominated option'. Other model outputs included total and incremental costs, total and incremental life years (LYs), total and incremental QALYs, ICERs $(€ / L Y)$ and incremental cost-utility ratio (ICUR; €/QALY).

\section{Sensitivity Analyses}

The robustness of the model was tested through 20 sensitivity scenarios (variations of nivolumab prices minus $2 \%$, minus $7 \%$, minus $20 \%$ and minus $50 \%$ from public price to address
CEESP requirement, survival distribution models, discounting, time horizon, rates of local and distant recurrences, distribution of subsequent therapies and utilities).

Specifically, a one-way DSA was undertaken by varying each parameter by its standard error, $95 \%$ CI or $\pm 10 \%$ of the expected value (base case) based on data availability. Parameters related to patient characteristics, treatment efficacy, survival hazard ratios, utility values, dosing, costs and resource use and adverse events were included in the one-way DSA.

PSA was used to assess the variation in the model results from the uncertainty around each parameter in the model. Model parameters were sampled from parametric distributions to generate 1000 estimates of the costs and effects in each treatment arm. A normal distribution was applied for costs and resource utilization parameters, and beta and Dirichlet distributions were used for utilities and probabilities. Multivariate normal distribution with correlation between curve parameters was used for the parametric survival models for RFS and OS. Lognormal distribution was applied for HRs [34]. Drug acquisition costs were exempt from the PSA.

\section{Compliance with Ethics Guidelines}

Ethical approval was not required as this article is based on previously conducted studies and does not contain any studies with human participants or animals performed by any of the authors.

\section{RESULTS}

\section{Indirect Comparisons}

Relative efficacy results of nivolumab and active comparators obtained from NMA and correlation equation are presented as HR $(95 \% \mathrm{CI})$ for RFS and OS in Online Resource Table 7. The HRs for both nivolumab and pembrolizumab versus observation showed significantly higher RFS and OS than low-dose interferon. Relative efficacy results in the subgroup with $B R A F$ 
mutation obtained from NMA and correlation equation (OS for nivolumab and pembrolizumab) are presented as HR (95\% CI) for RFS and OS in Online Resource Table 8. The HRs for nivolumab, pembrolizumab and dabrafenib plus trametinib versus observation showed significantly higher RFS and OS than low-dose interferon. For both populations, the base case analysis used a constant hazards assumption.

\section{Model Outputs}

RFS for adjuvant treatment with nivolumab and comparators was projected over the 20-year model time horizon (Fig. 2). This figure showed that nivolumab produced higher RFS than other comparators over the model time horizon. Figure 3 shows the efficiency frontier; observation, low-dose interferon and nivolumab were on the frontier, whereas pembrolizumab was dominated (i.e. lower QALYs and higher costs than nivolumab).

Table 1 presents total life years, QALYs and costs for each treatment. Nivolumab produced 9.53 LYs and 7.56 QALYs for a total cost of $€ 139,841$, and low-dose interferon produced 8.14 LYs and 6.41 QALYs for a total cost of
$€ 91,672$. Pembrolizumab produced 9.37 LYs and 7.53 QALYs for a total cost of $€ 154,133$. The ICUR for nivolumab versus low-dose interferon was $€ 37,886 / \mathrm{QALY}$ in the overall population, and pembrolizumab was dominated in the costeffectiveness analysis.

Table 2 presents the breakdown of costs for each comparator. Treatment costs represented $35 \%$ of total costs for nivolumab, $43 \%$ for pembrolizumab and $4 \%$ for low-dose interferon. Subsequent treatment costs (as a percentage of total costs) were higher in the observation arm than in the nivolumab arm (53\% versus $26 \%$, respectively), reflecting higher disease recurrence to advanced stages relative to nivolumab. Online Resource Fig. 1 shows the efficiency frontier for the subgroup with BRAF mutation. Table 3 presents total life years, QALYs and costs for each comparator in this subgroup. The ICUR for nivolumab versus low-dose interferon was $€ 37,871 / Q A L Y$. Pembrolizumab was dominated, and dabrafenib plus trametinib was under generalized dominance (i.e. ICUR for dabrafenib plus trametinib versus low-dose interferon was higher than the ICUR for nivolumab versus lowdose interferon) in the cost-effectiveness analysis for the subgroup with $B R A F$ mutation.

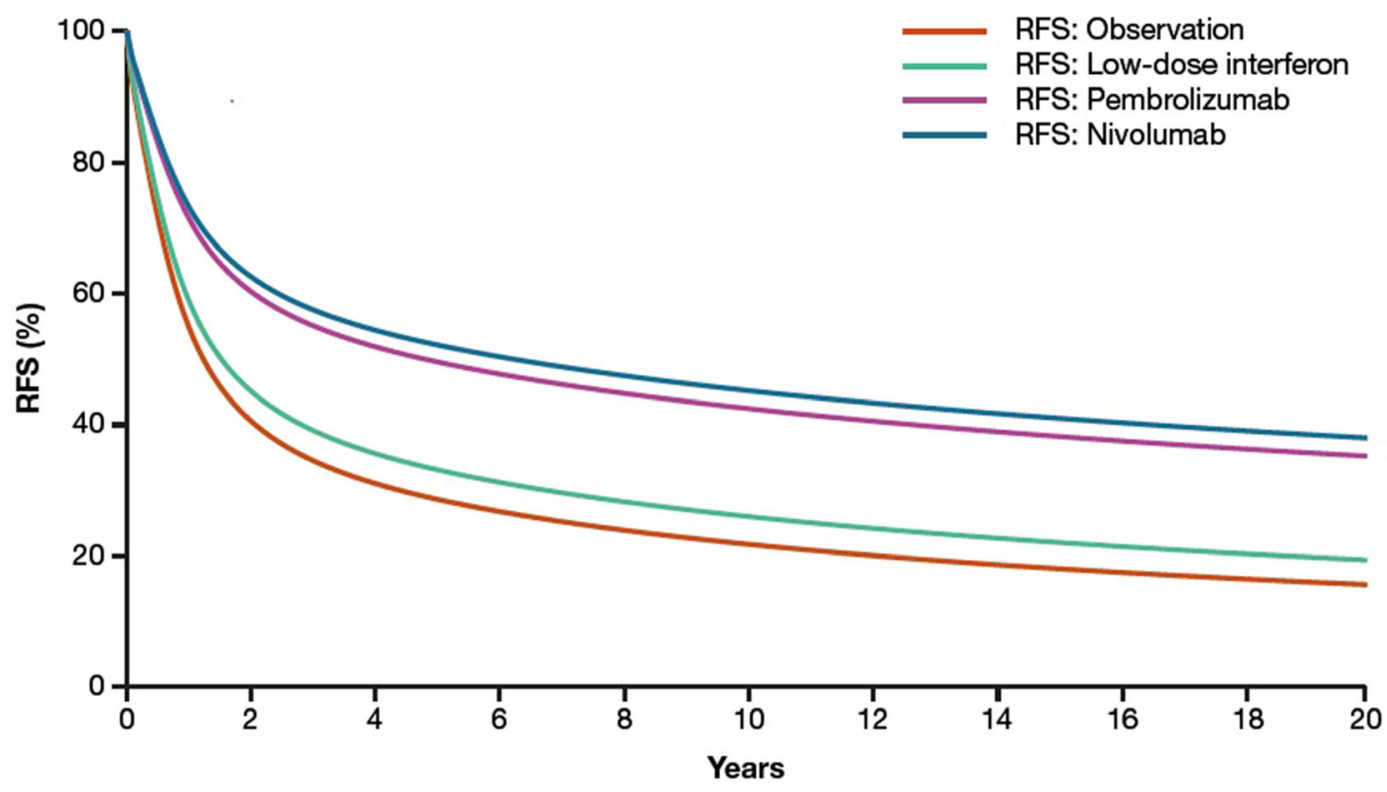

Fig. 2 RFS modelled for base case population (fixed effects HRs). HR hazard ratio, RFS recurrence-free survival 


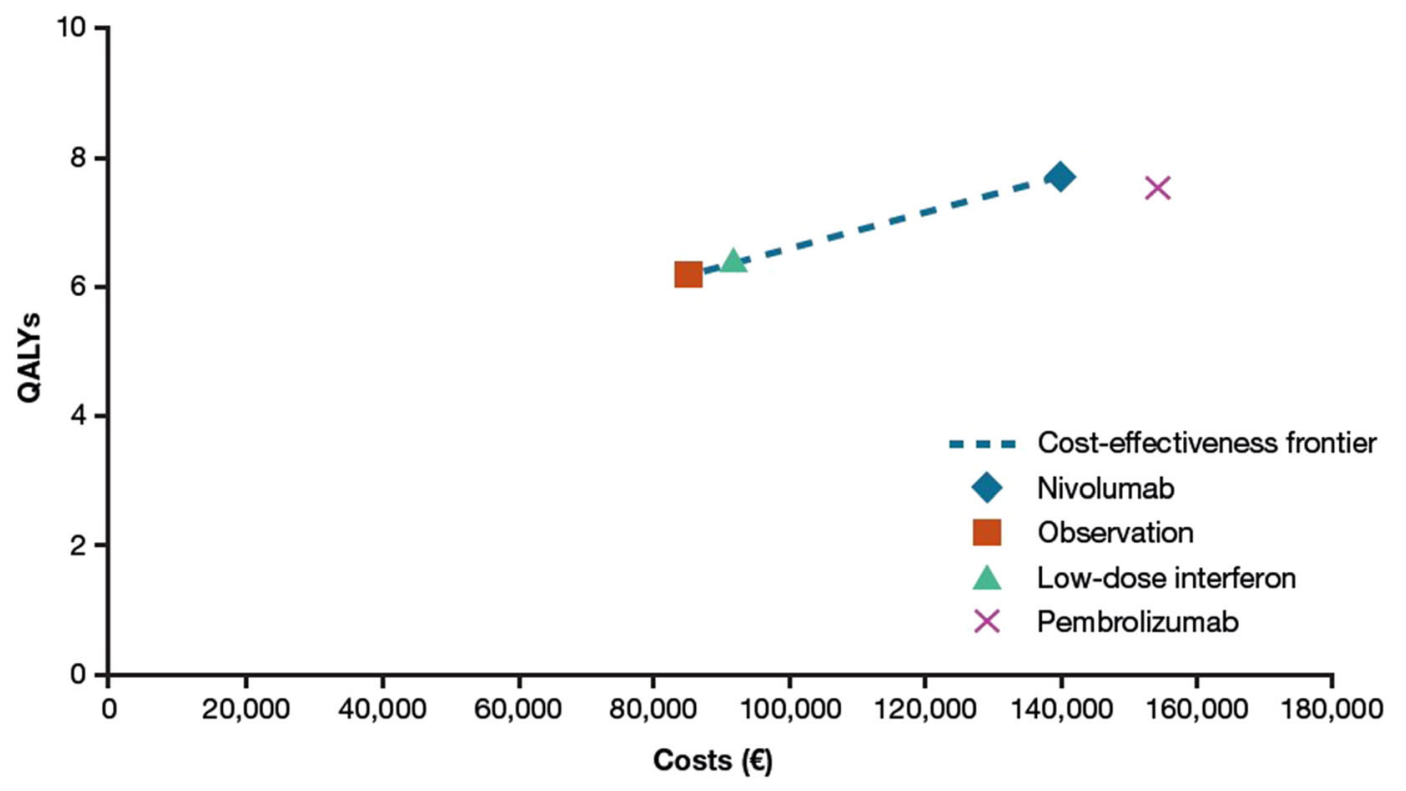

Fig. 3 Efficiency (cost-effectiveness) frontier for base case analysis in overall population. QALY quality-adjusted life year

Table 1 Cost-effectiveness results in overall population (model output)

\begin{tabular}{|c|c|c|c|c|c|c|c|c|}
\hline Comparator & $\begin{array}{l}\text { Total } \\
\text { costs }(€)\end{array}$ & $\begin{array}{l}\text { Total } \\
\text { LYs }\end{array}$ & $\begin{array}{l}\text { Total } \\
\text { QALYs }\end{array}$ & $\begin{array}{l}\text { Incremental } \\
\text { costs }(€)\end{array}$ & $\begin{array}{l}\text { Incremental } \\
\text { LYs }\end{array}$ & $\operatorname{ICER}(\boldsymbol{\epsilon} / \mathbf{L Y})$ & $\begin{array}{l}\text { Incremental } \\
\text { QALYs }\end{array}$ & ICUR (€/QALY) \\
\hline Observation & 85,011 & 7.84 & 6.18 & - & & & - & - \\
\hline $\begin{array}{l}\text { Low-dose } \\
\quad \text { interferon }\end{array}$ & 91,672 & 8.14 & 6.41 & 6661 & 0.30 & 21,983 versus observation & 0.23 & $\begin{array}{r}29,010 \text { versus } \\
\text { observation }\end{array}$ \\
\hline Nivolumab & 139,841 & 9.53 & 7.68 & 48,169 & 1.39 & 34,585 versus low-dose interferon & 1.27 & $\begin{array}{c}37,886 \text { versus low- } \\
\text { dose interferon }\end{array}$ \\
\hline Pembrolizumab & 154,133 & 9.37 & 7.53 & 14,293 & -0.17 & Dominated & -0.15 & Dominated \\
\hline
\end{tabular}

ICER incremental cost-effectiveness ratio, ICUR incremental cost-utility ratio, $L Y$ life year, $Q A L Y$ quality-adjusted life year

\section{One-Way DSA-Overall Population}

DSA results for nivolumab versus low-dose interferon are shown in a tornado diagram (Online Resource Fig. 2). Sensitivity analyses are presented with the 10 first parameters influencing the cost-utility results. The DSA indicated that the ICUR was most sensitive to parameters of constant HR for nivolumab versus observation in RFS and discount rate for QALYs, with ICUR values ranging from $€ 26,216 / \mathrm{QALY}$ to $€ 68,016 / \mathrm{QALY}$. The RFS HR is the biggest driver, as it is also used in the correlation equation to derive nivolumab and pembrolizumab OS.

\section{PSA-Overall Population}

Based on 1000 iterations of the PSA, the average incremental cost was $€ 46,542$ and the average incremental QALY gain was 1.27 for nivolumab versus low-dose interferon. The PSA resulted in a probabilistic ICUR of $€ 36,576 /$ QALY for nivolumab versus low-dose interferon, representing a 3\% deviation from the deterministic ICUR of $€ 37,886 /$ QALY.

Online Resource Fig. 3 shows the cost-effectiveness plane of nivolumab versus low-dose interferon. It shows that all iterations were found in the same quadrant. Figure 4 shows the cost-effectiveness acceptability curve (CEAC) for 
Table 2 Breakdown of costs for overall population (model output)

\begin{tabular}{lcccc}
\hline Cost $(\boldsymbol{\epsilon})$ & Observation & Low-dose interferon & Nivolumab & Pembrolizumab \\
\hline Treatment & 0 & 3978 & 48,707 & 65,996 \\
Administration & 0 & 1359 & 10,868 & 7295 \\
Monitoring $^{\mathrm{a}}$ & 35,405 & 36,275 & 40,716 & 40,228 \\
Adverse events $^{\text {Subsequent therapies }}$ & 0 & 2447 & 299 & 241 \\
Palliative care & 45,409 & 43,584 & 35,996 & 37,024 \\
Total & 4196 & 4029 & 3257 & 3349 \\
\hline
\end{tabular}

All costs correspond to 2019 values

${ }^{a}$ Monitoring costs included follow-up and transportation costs

all comparators. As shown by the CEAC, for a willingness-to-pay threshold of $€ 52,000 / \mathrm{QALY}$, nivolumab had a probability higher than $80 \%$ of maximizing the net benefit.

\section{DISCUSSION}

We estimated the cost-effectiveness of nivolumab and comparators from a French societal perspective. The analysis was performed in accordance with national guidelines, which required a comparative analysis taking into consideration all relevant comparators in an efficiency frontier approach [19]. As a result, the analysis was not limited to a standard pairwise comparison, and the primary outcome of the analysis, submitted to CEESP as part of the pricing and reimbursement process, therefore consisted of establishing an efficiency frontier.

Comparators were selected using national academic recommendations [25], which explained why only low-dose interferon was retained among the various forms of interferons, on top of observation and recently approved therapies for the adjuvant treatment of resected melanoma, i.e. nivolumab, pembrolizumab and also dabrafenib plus trametinib in patients with $B R A F$ mutation.

The results from the economic evaluation suggested that, over a 20-year time horizon, nivolumab was a cost-effective strategy for the treatment of adult patients with resected melanoma. In the overall population, therapeutic strategies on the efficiency frontier consisted of observation, low-dose interferon and nivolumab, whilst pembrolizumab was strictly dominated. The economic model produced for nivolumab resulted in 7.68 QALYs for a mean total cost of $€ 139,841$. The ICUR of nivolumab versus low-dose interferon (the therapeutic strategy closest on the efficiency frontier) was $€ 37,886 /$ QALY. Analyses conducted in other countries achieved lower ICUR levels that may be primarily explained by their pairwise approach versus observation [35-37].

In France, decisions for reimbursement are not based on a willingness-to-pay threshold. In the French health care system, the ICUR value is used as a component of the pricing negotiation. Indeed, earlier studies suggested a theoretical threshold at a level of three times the national gross domestic product (GDP) per capita, estimated around $€ 100,000 / Q A L Y$ [38]. The observed ICUR in the present study was far below this theoretical threshold.

This study used a partitioned survival modelling approach, which has become a standard in cost-effectiveness analyses in oncology, notably for estimating the health benefits of innovative therapies in the metastatic stages. Whether it was adapted for earlier stages, such as for the adjuvant treatment of surgically resected tumours could be subject to discussion. 
Table 3 Cost-effectiveness results in subgroup with $B R A F$ mutation (model output)

\begin{tabular}{|c|c|c|c|c|c|c|c|c|}
\hline Comparator & $\begin{array}{l}\text { Total } \\
\text { costs }(€)\end{array}$ & $\begin{array}{l}\text { Total } \\
\text { LYs }\end{array}$ & $\begin{array}{l}\text { Total } \\
\text { QALYs }\end{array}$ & $\begin{array}{l}\text { Incremental } \\
\text { costs }(\boldsymbol{\epsilon})\end{array}$ & $\begin{array}{l}\text { Incremental } \\
\text { LYs }\end{array}$ & $\operatorname{ICER}(€ / \mathbf{L Y})$ & $\begin{array}{l}\text { Incremental } \\
\text { QALYs }\end{array}$ & ICUR (€/QALY) \\
\hline Observation & 120,411 & 7.87 & 6.20 & & & & & \\
\hline $\begin{array}{l}\text { Low-dose } \\
\quad \text { interferon }\end{array}$ & 126,362 & 8.17 & 6.42 & 5951 & 0.30 & $\begin{array}{r}19,546 \text { versus } \\
\text { observation }\end{array}$ & 0.22 & $\begin{array}{r}26,845 \text { versus } \\
\text { observation }\end{array}$ \\
\hline Nivolumab & 169,381 & 9.40 & 7.56 & 43,018 & 1.23 & $\begin{array}{l}\text { 34,881 versus low- } \\
\text { dose interferon }\end{array}$ & 1.14 & $\begin{array}{c}37,871 \text { versus low- } \\
\text { dose interferon }\end{array}$ \\
\hline Pembrolizumab & 183,307 & 9.40 & 7.55 & 13,926 & 0.00 & Dominated & -0.01 & Dominated \\
\hline $\begin{array}{l}\text { Dabrafenib plus } \\
\text { trametinib }\end{array}$ & 293,234 & 9.84 & 7.78 & 109,927 & 0.43 & $\begin{array}{l}\text { Extendedly } \\
\text { dominated }\end{array}$ & 0.23 & $\begin{array}{l}\text { Extendedly } \\
\text { dominated }\end{array}$ \\
\hline
\end{tabular}

All costs correspond to 2019 values

ICER incremental cost-effectiveness ratio, ICUR incremental cost-utility ratio, $L Y$ life year, $Q A L Y$ quality-adjusted life year

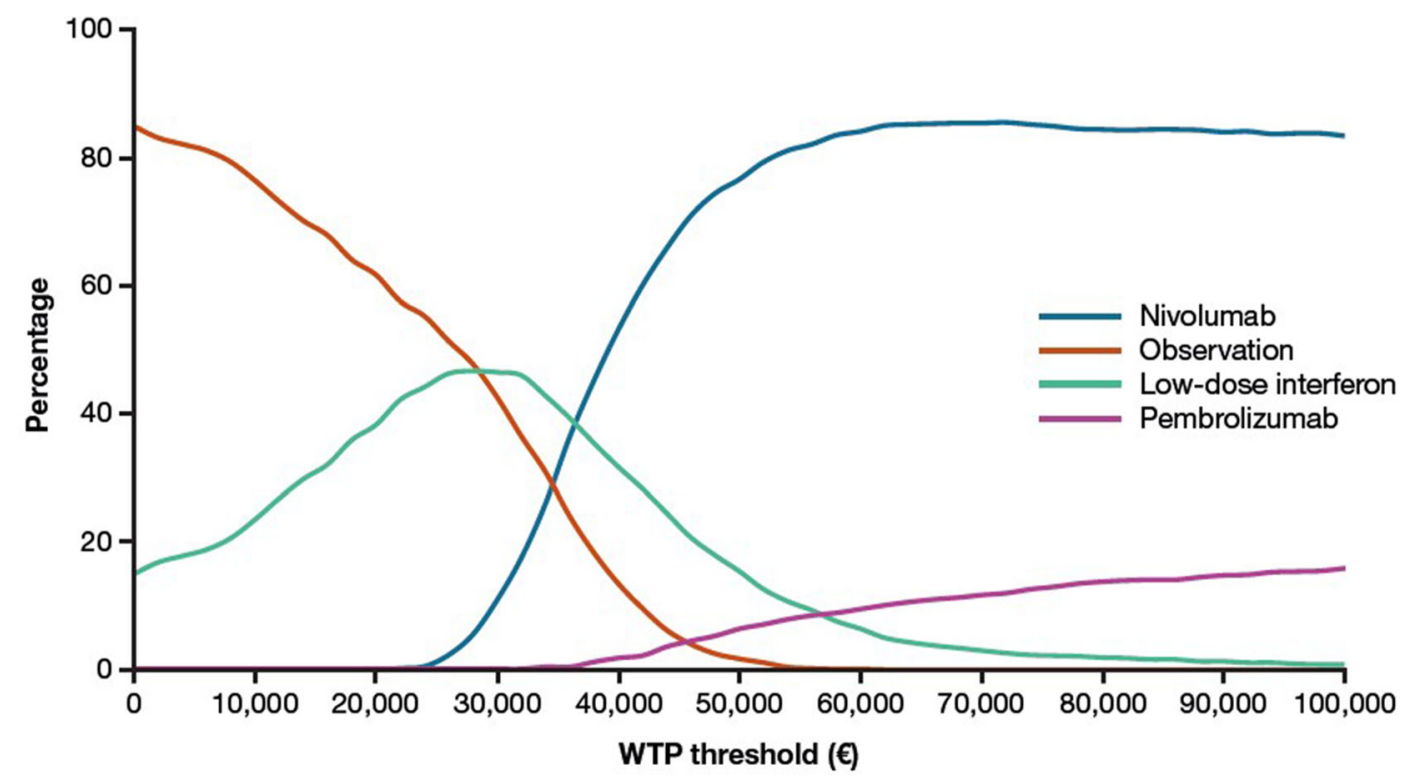

Fig. 4 Cost-effectiveness acceptability curve for all comparators. WTP willingness to pay

Indeed, another study recently published for the adjuvant treatment of melanoma [35] used a Markov state transition model. The choice between both modelling approaches was further reviewed by Smare et al. [39], using the treatment of metastatic renal cell carcinoma as reference, who concluded that differences in the assumptions underlying the choice of model structure are likely to produce different estimates of QALY gains. Finally, a more specific response to this question for the adjuvant treatment of resected melanoma was provided by some health technology assessment appraisals published in the UK by the National Institute for Health and Care Excellence [36] and in Scotland by the Scottish Medicines Consortium [37]. In these appraisals of nivolumab as adjuvant treatment of resected melanoma, both partitioned survival and Markov modelling approaches were reviewed; it appeared that the ICUR for nivolumab versus observation was similar, thus suggesting that the choice of 
model had limited impact on the outcome of the economic analysis.

Another key finding of this study was that nivolumab strictly dominated pembrolizumab. This could be explained by two factors. First, in terms of health benefits, the results for RFS estimated by the NMA reported a slightly more favourable HR for nivolumab versus observation than for pembrolizumab, with an estimated 8\% difference. A similar result was then found for OS, with the HRs versus observation projected through the correlation equation. Consequently, the model simulated more life years for nivolumab, with 2\% more QALYs (7.68 QALYs for nivolumab and 7.53 QALYs for pembrolizumab). Overall, both anti-PD-1s had very similar health benefits. Second, the treatment costs for pembrolizumab were higher than for nivolumab. As previously noted, these costs were the key driver of total costs, as all other costs were similar between the two anti-PD-1s; therefore, estimated mean total costs were $12 \%$ higher for pembrolizumab than for nivolumab.

In the subgroup with $B R A F$ mutation, dabrafenib plus trametinib combination therapy was added to the comparators. The components of the efficiency frontier were not changed. Dabrafenib plus trametinib was under extended dominance. In the analysis presented here, the indirect comparison used a constant hazards assumption, which appeared to be conservative for nivolumab. Dabrafenib plus trametinib had higher treatment costs than nivolumab. The ICUR of nivolumab versus low-dose interferon was $€ 37,871 / \mathrm{QALY}$, representing a negligible difference with the ICUR of nivolumab versus low-dose interferon in the overall population.

This study has several limitations. First, OS data were immature for nivolumab and pembrolizumab from the respective pivotal trials CheckMate 238 and KEYNOTE-054 and were not available for this analysis. This required use of surrogacy approaches, such as the correlation equation. This equation could, however, be validated externally by observing that it successfully predicted the $\mathrm{HR}_{\mathrm{OS}}$ of ipilimumab versus placebo in EORTC 18071 [7].

Immature survival data are common in studies conducted in earlier stages of cancer, and it is likely that with innovative treatment options for resected melanoma (and other tumours), the likelihood of having immature OS data, in either a clinical trial setting or reallife setting, will be increasing in the future. This highlights the need for further developing surrogate measures for economic analyses in early stages of cancer.

Another limitation is the uncertainty on key outcomes relative to the therapies used to treat patients with distant metastatic recurrences. One key question for which the medical community has few published recommendations to date is whether patients who receive an innovative adjuvant therapy for resected melanoma could be re-treated by the same therapy or the same therapeutic class after metastatic recurrence. Depending on the differences in the timing of local/regional recurrence, the clinician's choice of therapy after metastatic recurrence may impact OS.

The modelling approach used as reference for survival was extrapolated from the placebo arm of EORTC 18071 [7]. The re-treatment options available when this study was conducted may not reflect newer therapies used for metastatic melanoma.

Lastly, the national guidelines did not recommend the inclusion of indirect costs in the analysis; therefore, the impact on work productivity loss or caregiver burden is unknown. The analysis does, however, consider transportation cost for intravenous administration of therapies and for management of adverse events.

Further research is needed to estimate costeffectiveness of adjuvant therapies, in particular when OS data from clinical trials or the realworld setting are mature or available.

\section{CONCLUSIONS}

The results of this economic analysis suggest that, from a French societal perspective, nivolumab is a cost-effective treatment strategy in adult patients with resected melanoma. The ICUR for nivolumab versus low-dose interferon was $€ 37,886 / \mathrm{QALY}$ in the overall population. The therapeutic strategy of treating patients with nivolumab reached a probability of $80 \%$ of 
maximizing the net benefit of a willingness-topay threshold of $€ 52,000 / \mathrm{QALY}$.

\section{ACKNOWLEDGEMENTS}

Funding. This study was funded by BristolMyers Squibb Company, who is also funding the journal's Rapid Service and Open Access Fees.

Authorship. All named authors meet the International Committee of Medical Journal Editors (ICMJE) criteria for authorship for this article, take responsibility for the integrity of the work as a whole, and have given their approval for this version to be published.

Prior Presentation. Part of this work has been presented as a poster at International Society for Pharmacoeconomics and Outcomes Research (ISPOR), 2-6 November 2019, Copenhagen, Denmark.

Disclosures. Bruno Bregman, Anne-Françoise Gaudin, and François-Emery Cotté are employees of Bristol Myers Squibb. Adenike Amadi and Andriy Moshyk are employees and shareholders in Bristol Myers Squibb. Siguroli Teitsson, Isabella Orsini and Stéphane Roze have nothing to disclose.

Compliance with Ethics Guidelines. Ethical approval was not required as this article is based on previously conducted studies and does not contain any studies with human participants or animals performed by any of the authors.

Data Availability. Bristol Myers Squibb's policy on data sharing may be found at https:// www.bms.com/researchers-and-partners/indep endent-research/data-sharing-request-process. html.

Open Access. This article is licensed under a Creative Commons Attribution-NonCommercial 4.0 International License, which permits any non-commercial use, sharing, adaptation, distribution and reproduction in any medium or format, as long as you give appropriate credit to the original author(s) and the source, provide a link to the Creative Commons licence, and indicate if changes were made. The images or other third party material in this article are included in the article's Creative Commons licence, unless indicated otherwise in a credit line to the material. If material is not included in the article's Creative Commons licence and your intended use is not permitted by statutory regulation or exceeds the permitted use, you will need to obtain permission directly from the copyright holder. To view a copy of this licence, visit http://creativecommons.org/licenses/by$\mathrm{nc} / 4.0 /$.

\section{REFERENCES}

1. Giblin AV, Thomas JM. Incidence, mortality and survival in cutaneous melanoma. J Plast Reconstr Aesthet Surg. 2007;60(1):32-40.

2. Institut National du Cancer. 2017. Projection de l'incidence et de la mortalité par cancer en France métropolitaine en 2017. https://www.e-cancer.fr/ content/download/220859/3008146/file/Projection_ de_1_incidence_et_de_la_mortalite_en_France_metr opolitaine_en_2017_mel_20180108.pdf. Accessed June 6, 2020.

3. National Cancer Institute. Cancer stat facts: melanoma of the skin. https://seer.cancer.gov/statfacts/ html/melan.html. Accessed June 6, 2020.

4. NCCN Guidelines Version 2. 2018. Melanoma. https://www.nccn.org/professionals/physician_gls/ default.aspx\#site. Accessed June 6, 2020.

5. Eggermont AM, Suciu S, Santinami M, et al. Adjuvant therapy with pegylated interferon alfa- $2 b$ versus observation alone in resected stage III melanoma: final results of EORTC 18991, a randomised phase III trial. Lancet. 2008;372(9633): 117-26.

6. Ives NJ, Suciu S, Eggermont AMM, et al. Adjuvant interferon-alpha for the treatment of high-risk melanoma: an individual patient data meta-analysis. Eur J Cancer. 2017;82:171-83.

7. Eggermont AM, Chiarion-Sileni V, Grob JJ, et al. Prolonged survival in stage III melanoma with ipilimumab adjuvant therapy. $\mathrm{N}$ Engl J Med. 2016;375(19):1845-55. 
8. Eggermont AM, Chiarion-Sileni V, Grob JJ, et al. Adjuvant ipilimumab versus placebo after complete resection of high-risk stage III melanoma (EORTC 18071): a randomised, double-blind, phase 3 trial. Lancet Oncol. 2015;16(5):522-30.

9. Eggermont AMM, Blank CU, Mandala M, et al. Adjuvant pembrolizumab versus placebo in resected stage III melanoma. $\mathrm{N}$ Engl $\mathrm{J}$ Med. 2018;378(19):1789-801.

10. Long GV, Hauschild A, Santinami M, et al. Adjuvant dabrafenib plus trametinib in stage III BRAFmutated melanoma. N Engl J Med. 2017;377(19): 1813-23.

11. Weber J, Mandala M, Del Vecchio M, et al. Adjuvant nivolumab versus ipilimumab in resected stage III or IV melanoma. N Engl J Med. 2017;377(19): 1824-35.

12. Opdivo ${ }^{\circledR}$ EPAR Product Information. https://www. ema.europa.eu/en/medicines/human/EPAR/opdi vo\#product-information-section. Accessed June 6, 2020.

13. Keytruda ${ }^{\circledR}$ EPAR Product Information. https:// www.ema.europa.eu/en/medicines/human/EPAR/ keytruda\#product-information-section. Accessed June 6, 2020.

14. Mekinist ${ }^{\circledR}$ EPAR Product Information. https://www. ema.europa.eu/en/medicines/human/EPAR/mekini st\#product-information-section. Accessed June 6, 2020.

15. Bohensky MA, Pasupathi K, Gorelik A, et al. A costeffectiveness analysis of nivolumab compared with ipilimumab for the treatment of BRAF wild-type advanced melanoma in Australia. Value Health. 2016;19(8):1009-155.

16. Kohn CG, Zeichner SB, Chen Q, et al. Cost-effectiveness of immune checkpoint inhibition in BRAF wild-type advanced melanoma. J Clin Oncol. 2017;35(11):1194-202.

17. Oh A, Tran DM, McDowell LC, et al. Cost-effectiveness of nivolumab-ipilimumab combination therapy compared with monotherapy for first-line treatment of metastatic melanoma in the United States. J Manag Care Spec Pharm. 2017;23(6): 653-64.

18. Tarhini A, Benedict A, McDermott D, et al. Sequential treatment approaches in the management of BRAF wild-type advanced melanoma: a cost-effectiveness analysis. Immunotherapy. 2018;10(14):1241-52.

19. Haute Autorité de Santé. Choices in methods for economic evaluation, a methodological guide. https://www.has-sante.fr/upload/docs/application/ pdf/2012-10/choices_in_methods_for_economic_ evaluation.pdf. Accessed June 6, 2020.

20. Toumi M, Motrunich A, Millier A, et al. Analysis of health economics assessment reports for pharmaceuticals in France-understanding the underlying philosophy of CEESP assessment. J Mark Access Health Policy. 2017;5(1):1344088.

21. Langley PC. HAS should not be NICE: rejecting imaginary worlds in the French technology assessment guidelines. Innov Pharm. 2017;8(1).

22. Lanitis T, Cotte FE, Gaudin AF, et al. Stroke prevention in patients with atrial fibrillation in France: comparative cost-effectiveness of new oral anticoagulants (apixaban, dabigatran, and rivaroxaban), warfarin, and aspirin. J Med Econ. 2014;17(8): 587-98.

23. Chevalier J, Chamoux C, Hammes F, et al. Cost-effectiveness of treatments for relapsing remitting multiple sclerosis: a French societal perspective. PLoS ONE. 2016;11(3):e0150703.

24. Dummer R, Hauschild A, Lindenblatt $\mathrm{N}$, et al. Cutaneous melanoma: ESMO Clinical Practice Guidelines for diagnosis, treatment and follow-up. Ann Oncol. 2015;26(Suppl 5):v126-v132132.

25. Guillot B, Dalac S, Denis MG, et al. French updated recommendations in stage I-III melanoma treatment and management. J Eur Acad Dermatol Venereol. 2017;31(4):594-602.

26. Grange F, Mohr P, Harries M, et al. Economic burden of advanced melanoma in France, Germany and the UK: a retrospective observational study (Melanoma Burden-of-Illness Study). Melanoma Res. 2017;27(6):607-18.

27. Harries M, Mohr P, Grange F, et al. Treatment patterns and outcomes of stage IIIB/IIIC melanoma in France, Germany and the UK: a retrospective and prospective observational study (MELABIS). Int J Clin Pract. 2017;71(5):e12946.

28. Coart E, Suciu S, Saad E, et al. Prediction of overall survival benefit from relapse-free survival benefit of adjuvant nivolumab in completely resected melanoma. In: Presented at: 15th International Congress of the Society for Melanoma Research, October 24-27, 2018; Manchester, UK.

29. Suciu S, Eggermont AMM, Lorigan P, et al. Relapsefree survival as a surrogate for overall survival in the evaluation of stage II-III melanoma adjuvant therapy. J Natl Cancer Inst. 2018;110(1):87.

30. Burzykowski T, Molenbefghs G, Buyse M. The validation of surrogate end points by using data from 
randomized clinical trials: a case-study in advanced colorectal cancer. J R Stat Soc A. 2004;167(1): 103-24.

31. Burzykowski T, Molenbefghs G, Buyse M, et al. Validation of surrogate end points in multiple randomized clinical trials with failure time end points. J R Stat Soc C. 2001;50(4):405-22.

32. Latimer N. NICE DSU Technical Support Document 14: survival analysis for economic evaluations alongside clinical trials - extrapolation with patientlevel data. 2011. https://nicedsu.org.uk/wpcontent/uploads/2016/03/NICE-DSU-TSD-Survivalanalysis.updated-March-2013.v2.pdf. Accessed June 6, 2020.

33. Caro JJ, Nord E, Siebert U, et al. The efficiency frontier approach to economic evaluation of health-care interventions. Health Econ. 2010;19(10):1117-27.

34. Briggs AH, Claxton K, Sculpher MJ. Decision modelling for health economic evaluation. Oxford: Oxford University Press; 2006.

35. Bensimon AG, Zhou ZY, Jenkins M, et al. Cost-effectiveness of pembrolizumab for the adjuvant treatment of resected high-risk stage III melanoma in the United States. J Med Econ. 2019;22(10): 981-93.

36. NICE. January 23, 2019. Nivolumab for adjuvant treatment of completely resected melanoma with lymph node involvement or metastatic disease. Technology appraisal guidance. https://www.nice. org.uk/guidance/ta558. Accessed June 6, 2020.

37. Scottish Medicines Consortium. SMC 2112. Final advice, 9th November 2018 https://www. scottishmedicines.org.uk/media/3958/nivolumabopdivo-final-nov-2018-for-website.pdf. Accessed June 6, 2020.

38. Chouaid C, Bensimon L, Clay E, et al. Cost-effectiveness analysis of pembrolizumab versus standard-of-care chemotherapy for first-line treatment of PD-L1 positive (>50\%) metastatic squamous and non-squamous non-small cell lung cancer in France. Lung Cancer. 2019;127:44-52.

39. Smare C, Lakhdari K, Doan J, et al. Evaluating partitioned survival and Markov decision-analytic modelling approaches for use in cost-effectiveness analysis: estimating and comparing survival outcomes. Pharmacoeconomics. 2020;38(1):97-108. 